УДК 115; 514; 517.95; 517.96

DOI $10.21661 / r-498027$

\title{
А.В. Витко
}

\section{ОБЪЕМНЫЕ СВОЙСТВА МНОГОМЕРНЫХ ОБЪЕКТОВ}

Аннотация: с иелью освоения трудных для восприятия моделей многомерности, рассматриваются представления и технология (аппарат) описания и измерения объема и количества вещества, заполняющего объем многомерных объемных объектов. Сопоставляются альтернативные варианты многомерной совокупности одномерных моделей и единой многомерной модели объемных объектов. Показывается технология измерения многомерного объема и количества вещества, заполняющего объем, с выводом о том, что при переходе от множества координатных векторов материальных точек в полном телесном угле $4 \pi$ стерадиан к многомерной модели попарно ортогональных векторов вычисление объема многомерного объекта существенно упрощается. Приводится вывод о том, что потенциально бесконечномерная модель объекта представляет собой сферу, в которой объем объема, количество и энергия вещества, заполняющего объем, целиком сосредоточены на ее поверхности.

Ключевые слова: многомерные модели, объемные объекты, количество и энергия вещества, потенциальная бесконечность.

\section{A.V. Vitko}

\section{BULK PROPERTIES OF MULTIDIMENSIONAL OBJECTS}

Abstract: for the purpose of learning multidimensional models, difficult for perception, representations and technology (device) of the description and measurement of volume and amount of the substance filling the volume of multidimensional objects are considered. Alternative options of multidimensional set of one-dimensional models and unified multidimensional model of three-dimensional objects are compared. The technology of measurement of multidimensional volume and amount of the substance that fills volume is shown. The authors came to the conclusion when transferring from 
a set of coordinate vectors of material points in complete spatial angle $4 \pi$ steradian to multidimensional model of in pairs orthogonal vectors, calculation of volume of a multidimensional object significantly becomes simpler. The conclusion is that potentially infinite-dimensional model of an object represents the sphere in which balk of volume, the quantity and energy of the substance filling volume are entirely concentrated on its surface.

Keywords: multidimensional models, three-dimensional objects, quantity and energy of substance, potential infinity.

Человеку психологически очень трудно выйти за пределы привычных пространственных трех измерений. Многомерность, не имея зрительного образа, трудно постигается человеком. Что касается бесконечномерных пространств, то, хотя они во многом и аналогичны конечномерным, могут обладать некоторыми совершенно иными свойствами [5, с. 566].

Задача статьи состоит в достижении цели обеспечения освоения трудных для восприятия моделей многомерности, путем формирования представлений и технологии (аппарата) описания и измерения объема и количества вещества, заполняющего объем, в многомерных объемных объектах [2, с. 32-42]. В постановке и решении задачи учтен также фактор скорости изменения объема и количества, поскольку линейность пространства проще воспринимается, если объемы создаются путем изменения (расширения или сжатия) пространственного положения оболочек с линейными скоростями.

Представление объема и процесса его изменения как совокупности отдельных граничных материальных точек и их движения с линейными скоростями является основой современной технологии такого описания. Результатом при этом является не единая многомерная модель объема и количественных изменений, а совокупность одномерных моделей.

Так, применительно к двумерному варианту его «объем» представляется совокупностью трех точек, а его изменение - движением двух точек $k$ и $m$ с линейными скоростями $v_{K}$ и $v_{M}$, в результате чего образуется скоростной треугольник 
$0 \mathrm{~km}$, формирующий расширяющийся «объем», внешней границей которого является относительный путь $K M$ (рис. 1).

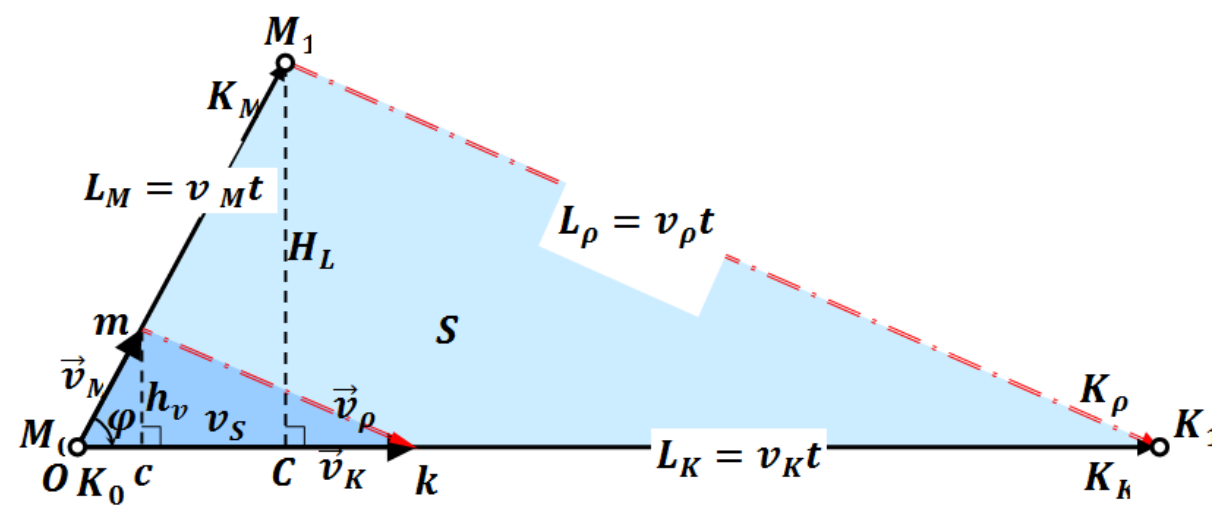

Рис. 1. Движение системы двух материальных точек из одной точки О

Количественной мерой «объема» и его изменения в результате движения этих точек являются расстояния, которые при равномерном прямолинейном движении точек характеризуется системой уравнений:

$$
\left.\begin{array}{l}
L_{K}=v_{K} t ; \\
L_{M}=v_{M} t .
\end{array}\right\}
$$

Совокупность одномерных линейных моделей (1) формирует двумерную модель - треугольник, количественной мерой «объема» которого является площадь

$$
S=\frac{1}{2} L_{K} L_{M} \sin \varphi
$$

Стороны треугольника (1) и его площадь (2) с учетом их изменений являются примером альтернативного представления двумерного объема совокупностью одномерных моделей и единой многомерной моделью.

Применительно к трехмерному варианту его объем представляется совокупностью четырех точек, а его изменение - движением трех точек $k, m$ и $n$, не принадлежащих одной плоскости, с линейными скоростями $v_{K}, v_{M}$ и $v_{N}$, в результате чего образуется объемная фигура - треугольная пирамида, внешней границей которой, является треугольник относительных путей $M K N$ (рис. 2). 


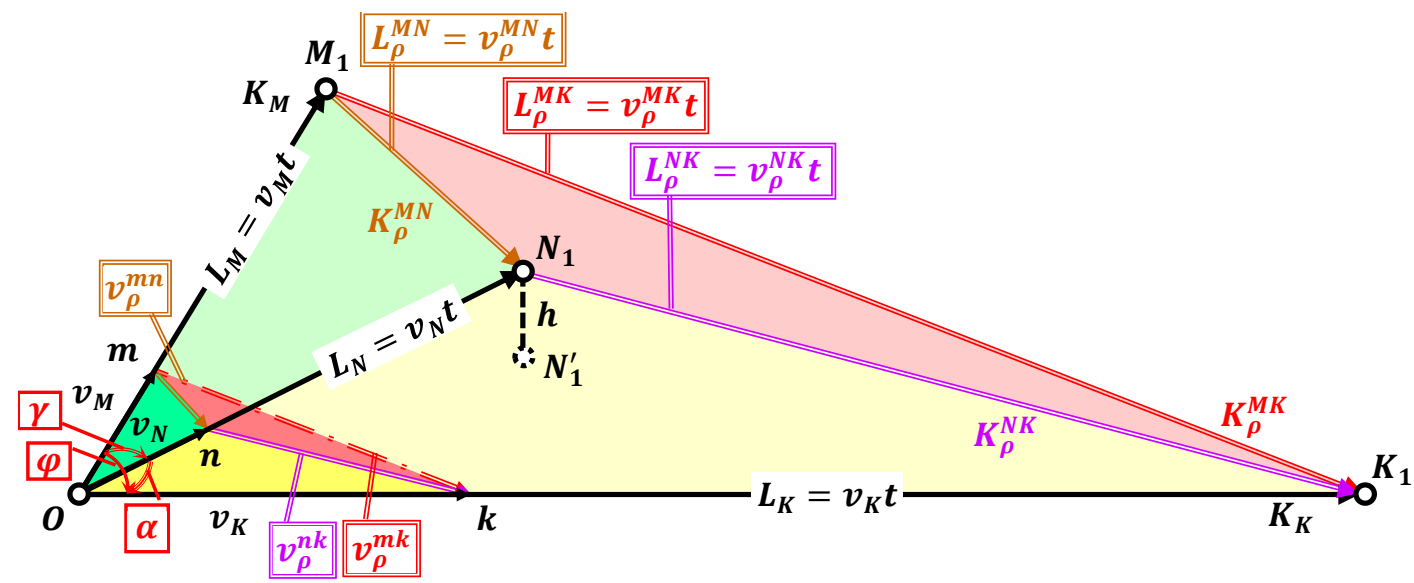

Рис. 2. Движение системы трех материальных точек из одной точки О

Количественной мерой объема и его изменения в результате движения этих точек являются расстояния, которые при равномерном прямолинейном движении точек характеризуется системой уравнений:

$$
\left.\begin{array}{l}
L_{K}=v_{K} t ; \\
L_{M}=v_{M} t ; \\
L_{N}=v_{N} t .
\end{array}\right\}
$$

Совокупность одномерных линейных моделей (3) формирует трехмерную модель - пирамиду, количественной мерой объема которой является объем [1, с. 187]:

$$
S^{2}=\frac{1}{288}\left|\begin{array}{ccccc}
0 & K_{K M}^{2} & K_{K N}^{2} & L_{K}^{2} & 1 \\
K_{K M}^{2} & 0 & K_{M N}^{2} & L_{M}^{2} & 1 \\
K_{K N}^{2} & K_{M N}^{2} & 0 & L_{N}^{2} & 1 \\
L_{K}^{2} & L_{M}^{2} & L_{N}^{2} & 0 & 1 \\
1 & 1 & 1 & 1 & 0
\end{array}\right| .
$$

Ребра пирамиды (3) и ее объем (4) являются примером альтернативного представления трехмерного объема совокупностью одномерных моделей и единой многомерной моделью.

Как итог, в результате движения с линейными скоростями две материальные точки образуют треугольник, три материальные точки, не принадлежащие одной плоскости, образуют треугольную пирамиду, четыре материальные точки, не принадлежащие одной плоскости, образуют - четырехугольную пирамиду и т. д. Поверхность, натянутая на окончания векторов перемещения отдельных материальных точек с различными скоростями, будет иметь пирамидальный вид, по 
мере неограниченного увеличения количества материальных точек принимающий игольчатый тип. Если же принять условие равенства линейных скоростей материальных точек, то системное движение из одной точки бесконечно возрастающего множества таких материальных точек в полном телесный угле $4 \pi$ стерадиан будет стремиться к сферической форме.

Итак, получен важный методологический вывод, состоящий в том, что бесконечномерная совокупность одномерных линейных моделей, количественно отражающих объемы многомерных объектов, при преобразовании этой совокупности в единую бесконечномерную модель объекта представляет собой сферу.

Что касается решения практической задачи - измерения многомерных объемов, то рассмотренный подход является неприемлемым по двум причинам: вопервых, как это видно из сравнения (2) и (4), очевидно возрастание сложности вычисления многомерных объемов по мере увеличения размерности; во-вторых, затруднительно сформировать модель многомерного объекта произвольной формы с помощью многомерных пирамид, которые строятся из одной точки.

По этим причинам вместо произвольно ориентированных векторов следует взять попарно ортогональные векторы единичной длины $e_{i}, \quad(i=1, \ldots, n)$. Если на них натянуть $n$-мерный скоростной кубик с модулем скорости $v$, то, с учетом текущего времени $t$, будет получен «путевой» кубик с ребрами длины $v t$. При $n=1$ такой многомерный объект будет представлять собой отрезок, при $n=2-$ квадрат, при $n=3$ - трехмерный куб, при произвольном $n-n$-мерный куб. Его текущий объем, естественно, равен $V_{n}=(v t)^{n}$. Как видно из приведенных сведений, переход от множества координатных векторов материальных точек в полном телесный угле $4 \pi$ стерадиан к многомерной модели попарно ортогональных векторов, вычисление объема многомерного объекта существенно упрощается. Таким образом, первый недостаток моделирования многомерного пространства устраняется.

Что касается второго недостатка, то в практических задачах измерения объемов объектов произвольной формы, отличающеюся от кубической, пользуются 
технологией интегрирования. Для многомерной модели заданного объекта можно построить из одномерных взаимно ортогональных моделей множество многомерных кубиков той же размерности с произвольно заданными линейными размерами ребер. Объем любой $n$-мерной фигуры произвольной формы можно определить, заполнив ее большим числом маленьких $n$-мерных кубиков так, чтобы они занимали внутренний объем фигуры максимальным образом, и затем сложив их объемы. При этом около поверхности $n$-мерной фигуры неизбежно останутся некоторые пустоты. Уменьшая размер граней $n$-мерных кубиков, то есть делая их все более мелкими, можно добиться сколь угодно точного заполнения объема $n$-мерной фигуры без приграничных пустот. Общий объем $n$-мерной фигуры будет равен сумме объемов всех наполняющих ее кубиков. В этом состоит технология интегрирования, которая применена здесь для измерения объемов в многомерных объемных объектах.

Пока речь шла о пустотелой конструкции многомерного объемного объекта. Задача усложняется, если мера «количества» отличается от объема, например, если мерой количества является масса. Тогда количественные изменения массы будут обусловливаться как ростом, увеличением объема объекта, так и $n$-мерной плотностью наполняющей объект среды, которая в районе $n$-мерной точки $x$ описывается функцией $f(x)$. «Количество» этой среды, например, масса, определяется суммой ее количеств во всех n-мерных кубиках заполнения объема объекта. Вся сумма «количества» есть интеграл Римана - одно из важнейших понятий математического анализа [3, с. 126, 127]:

$$
G=\lim \sum_{i} f\left(x_{i}\right) \Delta x_{i}=\int_{\Omega} f(x) \Delta x,
$$

где $\Omega-n$-мерный объем объекта.

Размерность $n$ может быть как угодно велика и даже бесконечна. В строгом математическом тексте определение бесконечномерности нужно вводить отдельно либо как актуальной, либо как потенциальной бесконечности. При рассуждениях, подобных приведенным выше, пользуются понятием актуальной бесконечности как неопределенно большой конечномерности. 
Примечание. Актуальная бесконечность - это понятие о бесконечной совокупности предметов определенного класса, задание которой завершено и предметы которой представлены одновременно в виде готового, актуально существующего множества очень большой конечной размерности.

Потенциальная бесконечность - это величина, которая все время возрастает, но никогда не достигает какого-либо определенного значения.

Все это относится к конечномерным объектам пространства заданной размерности, начиная с евклидового трехмерного пространства, привычного для обыденного восприятия, и кончая актуальной бесконечностью. Что касается неограниченного роста размерности пространства, то есть перехода к потенциальной бесконечности с одновременным переходом от бесконечномерной совокупности одномерных линейных моделей к единой бесконечномерной модели, то смысл такого перехода состоит в следующем.

Уменьшим модуль скорости всех материальных точек вдвое. Длина отрезка одномерной модели будет составлять $d=0.5$ от длины всего отрезка. Квадрат двумерной плоской модели будет иметь площадь 0.25 площади исходного квадрата, а площадь оставшегося квадратного кольца - 0.75 площади исходного квадрата. Куб с ребром $d=0.5$ от исходного будет иметь объем 0.125 объема исходного, а объем оставшегося кубического слоя будет составлять уже 0.875 объема исходного куба. Объем $n$-мерного куба с ребром $d=0.5$ от исходного должен выражаться формулой $d^{n}$. Поэтому доля объема куба с ребром $d=0.5$ будет составлять $(0,5)^{\Pi}$, стремясь к нулю вместе с ростом $n$. Десятимерный куб с ребром $d=0.5$ будет иметь объем 0.001, а оставшийся $n$-мерный кубический слой, соответственно, объем 0.999 от исходного.

Что важно, многомерный куб по мере увеличения размерности будет стремиться к сферической форме, как и в случае рассмотренной выше модели системы бесконечно возрастающего множества координатных векторов материальных точек в полном телесный угле $4 \pi$ стерадиан. Этот подход позволяет сформулировать геометрический образ потенциально бесконечномерного объекта: его объем целиком сосредоточен на его поверхности. 
Что касается варианта задачи, связанной с определением меры не объема, а количества, то вывод, соответственно, приобретает форму утверждения: «количество», в частном случае масса потенциально бесконечномерного объекта целиком сосредоточена на его поверхности.

Согласно [4, с. 14], асимптотические свойства многомерных объемов распространяются и на энергии, вследствие чего сосредоточенность объема и «количества» (энергии) многомерного тела на его поверхности, в сущности, предопределяет существование температуры как макроскопической величины.

Асимптотические свойства многомерных объемов - это геометрический арсенал статистической физики [4, с. 15]. В потенциальной бесконечномерности, где почти вся область - это ее граница, чтобы найти правильные способы думать и вычислять, нужны самые рафинированные орудия математического арсенала.

Когда рассматривается множество функций с числовыми значениями, то допустимо считать, что оно лежит в состоящем из векторов $n$-мерном вещественном числовом линейном банаховом пространстве с введённой на нём нормой [5, с. 644]. Под евклидовым обычно понимают конечномерное вещественное пространство $R^{n}$ со скалярным произведением. Гильбертово пространство $C^{n}$, обычно предполагающееся бесконечномерным, представляет собой обобщение евклидового, в котором координаты принимают комплексные значения [5, с. 452]. Так вот, согласно [5] рассмотренные выше свойства многомерных объектов распространяются и на гильбертово пространства, вследствие чего используются в теоретической радиоэлектронике, классической космографии, квантовой механике.

В целом, статья содержит краткое введение в физико-математическое представление и философское осмысление фрагмента теории многомерных пространств, содержащего переход от привычной трехмерности пространства к его бесконечномерности, где почти вся область - это граница ее поверхности, и где, чтобы найти правильные способы думать и вычислять, нужны самые рафинированные орудия математического арсенала. 


\section{Список литературы}

1. Бронштейн И.М. Справочник по математике для инженеров и учащихся втузов / И.М. Бронштейн, К.А. Семендяев. - М.: Наука, 1986. - 544 с.

2. Витко А.В. Комплементарность. Физика, математика, философия: монография. - СПб., 2019. - 160 с.

3. Корн Г. Справочник по математике: для научных работников и инженеров / Г. Корн, Т. Корн. - М., 1974. - 832 с.

4. Мании Ю.И. Математика и физика. - М.: Знание, 1979. - 64 с.

5. Пенроуз Р. Путь к реальности, или законы, управляющие вселенной: полный путеводитель / пер. с англ. А.Р. Логунова и Э.М. Эпштейна. - М.; Ижевск, 2007. $-911 \mathrm{c}$.

\section{References}

1. Bronshtein, I. M., \& Semendiaev, K. A. (1986). Spravochnik po matematike dlia inzhenerov i uchashchikhsia vtuzov., 544. M.: Nauka.

2. Vitko, A. V. (2019). Komplementarnost'. Fizika, matematika, filosofiia., 160. $\mathrm{SPb}$.

3. Korn, G., \& Korn, T. (1974). Spravochnik po matematike: dlia nauchnykh rabotnikov i inzhenerov., 832. M.

4. Manii, Iu. I. (1979). Matematika i fizika., 64. M.: Znanie.

5. Penrouz, R. (2007). Put' k real'nosti, ili zakony, upravliaiushchie vselennoi: polnyi putevoditel'., 911. M.;; Izhevsk.

Витко Александр Викторович - канд. физ.-мат. наук, руководитель, ФГКВОУ ВО «Военный учебно-научный центр Военно-Морского Флота «Военно-морская академия имени Адмирала Флота Советского Союза Н.Г. Кузнецова», Санкт-Петербург, Россия.

Vitko Aleksandr Viktorovich - candidate of physical and mathematical sciences, head, FSOMEI of HE "Military academic and research center of naval forces "N.G. Kuznetsov Naval Academy", Saint Petersburg, Russia. 
Научный руководитель Поленин Владимир Иванович - канд. техн. наук, д-р воен. наук, профессор, ФГКВОУ ВО «Военный университет» Минобороны России, Москва, Россия.

Scientific supervisor Polenin Vladimir Ivanovich - candidate of engineering sciences, doctor of military sciences, professor, FSOMEI of HE "Military University" of the Ministry of Defense of Russian Federation, Moscow, Russia. 